Original Article

\title{
Vincristine, Irinotecan, and Temozolomide as a Salvage Regimen for Relapsed or Refractory Sarcoma in Children and Young Adults
}

\author{
Hee Young Jü ${ }^{1,2}$, Meerim Park², Jun Ah Lee ${ }^{2}$, Hyeon Jin Park², Seog Yun Park ${ }^{3}$, June Hyuk Kim ${ }^{4}$ Hyun Guy Kang ${ }^{4}$, Hee Chul Yang ${ }^{5}$, \\ Byung-Kiu Park ${ }^{2}$ \\ ${ }^{1}$ Department of Pediatrics, Samsung Medical Center, Seoul, ${ }^{2}$ Center for Pediatric Oncology, National Cancer Center, Goyang, ${ }^{3}$ Department of Pathology, \\ National Cancer Center, Goyang, ${ }^{4}$ Orthopaedic Oncology Clinic, National Cancer Center, Goyang, ${ }^{5}$ Center for Lung Cancer, Research Institute \\ and Hospital, National Cancer Center, Goyang, Korea
}

Purpose No standard salvage regimen is available for relapsed or refractory sarcoma. We investigated the efficacy and toxicity of the vincristine, irinotecan, and temozolomide combination (VIT) for relapsed or refractory sarcomas of variable histology in children and young adults.

Materials and Methods We retrospectively reviewed data from the relapsed or refractory sarcoma patients who were treated with VIT. The VIT protocol was given every 3 weeks as follows: vincristine, $1.5 \mathrm{mg} / \mathrm{m}^{2}$ intravenously on day 1 , irinotecan, $50 \mathrm{mg} / \mathrm{m}^{2} / \mathrm{day}$ intravenously on days $1-5$, and temozolomide, $100 \mathrm{mg} / \mathrm{m}^{2} /$ day orally on days $1-5$.

Results A total of 26 patients (12 males) with various sarcoma histology were included in the study. Most common diagnosis was rhabdomyosarcoma $(n=8)$ followed by osteosarcoma $(n=7)$. Median age at the start of VIT was 18.5 years (range, 2.0 to 39.9). VIT was delivered as 2 nd to 7 th line of treatment, with 4 th line most common (9/26, 34.6\%). Median number of VIT courses given was 3 (range, 1 to 18). Of the 25 evaluable patients, there was two partial response (PR) and 11 stable disease (SD) with an overall control rate (complete remission+PR+SD) of 52\%. PR was seen in one (50\%) of the two evaluable patients with Ewing sarcoma and one (14.3\%) of the seven patients with osteosarcoma. Overall survival and progression-free survival rates were $79.3 \%$ and $33.9 \%$ at 1 year, and $45.5 \%$ and $25.4 \%$ at 2 years, respectively. There was no treatment-related mortality.

Conclusion The VIT regimen was effective and relatively safe in our cohort of sarcoma patients.

Key words Salvage therapy, Sarcoma, Vincristine, Temozolomide, Irinotecan

\section{Introduction}

Sarcoma is a group of disorder containing various histologic types of tumors, which arise from mesenchymal tissue. It is largely divided into bone and soft tissue sarcoma. Malignant bone and soft tissue sarcoma account for $6 \%$ and $7 \%$ of all cancers occurring under age 20, respectively $[1,2]$.

Outcome of sarcoma has improved significantly during the last several decades, and the adjusted 5-year survival rates for both bone and soft tissue in the United States and Korea were reported to be $71 \%-77 \%[3,4]$. However, recurrent or refractory patients fare much less, with 5 -year overall survival (OS) rate of $<40 \%$ [3]. Attempts have been made to obtain a better survival for relapsed or refractory sarcoma. Several salvage regimens, including the combination of ifosfamide, carboplatin, and etoposide (ICE) [5]; topotecan and cyclophosphamide (TC) [6]; docetaxel and gemcitabine [7]; irinotecan and temozolomide with vincristine (VIT) or without vincristine (IT) $[8,9]$ have been employed. Several studies testing VIT have revealed an enthusiastic response rate of $29 \%-63 \%$ in recurrent or refractory Ewing sarcoma [8-10]. However, reports on VIT trial are restricted mostly to Ewing sarcoma and rhabdomyosarcoma of pediatric age. Under this background, we wanted to explore the usefulness of the VIT regimen in children and young adults with various sarcoma histology.

Irinotecan is a camptothecin prodrug that is converted into SN-38 in vivo and acts as a topoisomerase I poison. Topoisomerase I inhibitors stabilize the enzyme-DNA covalent complex and cause S-phase specific cytotoxicity. Temozolomide is an oral alkylating agent. DNA damage from alkylators increases the sensitivity to topoisomerase I inhibitors. Schedule-dependent synergy between temozolomide and irinotecan has been shown in preclinical studies when temozolomide was given at least 1 hour before irinotecan administration [11]. Another advantage of these two-drug combination is that they have non-overlapping toxicities and differing mechanisms of resistance [12]. In addition, vincris-

Correspondence: Byung-Kiu Park

Center for Pediatric Oncology, National Cancer Center, 323 Ilsan-ro, Ilsandong-gu, Goyang 10408, Korea

Tel: 82-31-920-1240 Fax: 82-31-920-1244 E-mail: bkpark@ncc.re.kr

Received February 3, 2021 Accepted June 11, 2021 Published Online June 14, 2021 
tine is known to show synergistic effect with irinotecan and temozolomide in rhabdomyosarcoma and Ewing sarcoma [13].

Herein, we describe the antitumor response, survival outcome, and toxicity experienced with VIT retrospectively collected in our patients with relapsed or refractory sarcoma.

\section{Materials and Methods}

\section{Patient population and data collection}

We retrospectively reviewed the medical records of relapsed or refractory sarcoma patients who were treated with the VIT regimen at the Center for Pediatric Oncology of the National Cancer Center (NCC), Korea between 2012 and 2018. Histologic diagnosis was made by an expert in sarcoma pathology. The following data were collected: age at initial diagnosis and VIT therapy, sex, location of tumor, information on prior chemotherapy, number of relapses, time to recurrence after initial treatment, local control strategy, status at last follow-up, and follow-up period.

\section{Treatment}

The VIT protocol given every 3 weeks was as follows: vincristine, $1.5 \mathrm{mg} / \mathrm{m}^{2}$ intravenously on day 1 , irinotecan, 50 $\mathrm{mg} / \mathrm{m}^{2} /$ day intravenously on days $1-5$, and temozolomide, $100 \mathrm{mg} / \mathrm{m}^{2} /$ day orally on days $1-5$. Temozolomide was administered at least 1 hour before irinotecan. Prophylactic cefixime at $4 \mathrm{mg} / \mathrm{kg} /$ day orally was prescribed to prevent or reduce irinotecan-associated diarrhea according to previous reports [14]. Loperamide with or without atropine was given if patients developed diarrhea. Tests for blood counts, and renal and liver function were taken before each course and then weekly, but more frequently if clinically indicated. An imaging work-up for extent of disease was performed before VIT initiation, every two to three courses, or any time tumor progression was suspected clinically. It included computed tomography, magnetic resonance imaging, bone scan, or positron emission tomography scans. VIT was withdrawn (VIT failure) with disease progression or at physician's discretion even with sustained stable disease (SD) or occurrence of unacceptable toxicities. Maximum number of VIT courses were 18 , covering neoadjuvant and adjuvant phases. Patients who received at least two courses were evaluated for efficacy and toxicity was assessed for those who completed at least one course. Treatment response was assessed according to Response Evaluation Criteria in Solid Tumor ver. 1.1 criteria [15]. Best overall response was defined as the best response recorded from the start of treatment until disease progression or recurrence.

Surgery or radiotherapy was given depending on individ-
Table 1. Patient characteristics

\begin{tabular}{|cc}
\hline Characteristic & No. $(\%)(\mathbf{n}=\mathbf{2 6})$ \\
\hline Age at diagnosis, median (range, yr) & $14.9(1.0-35.2)$ \\
\hline Age at VIT initiation, median (range, yr) & $18.5(2.0-39.9)$ \\
Sex & \\
\hline Male & $12(46.2)$ \\
\hline Female & $14(53.8)$ \\
\hline Diagnosis & \\
\hline Rhabdomyosarcoma & $8(30.8)$ \\
\hline Osteosarcoma & $7(26.9)$ \\
\hline Ewing sarcoma & $3(11.5)$ \\
\hline Synovial sarcoma & $3(11.5)$ \\
\hline Alveolar soft part sarcoma & $2(7.7)$ \\
\hline High grade sarcoma) & $1(3.8)$ \\
\hline Hemangiopericytoma & $1(3.8)$ \\
\hline Desmoplastic small round cell tumor & $1(3.8)$ \\
Tumor status at VIT initiation & \\
\hline No evidence of disease & $1(3.8)$ \\
\hline Gross tumor & $25(96.2)$ \\
Primary disease at VIT initiation & $4(15.4)$ \\
\hline Metastatic disease at VIT initiation & $21(80.8)$ \\
VIT treatment line & $2(7.7)$ \\
\hline 2 & $5(19.2)$ \\
\hline 3 & $9(34.6)$ \\
\hline 4 & $4(15.4)$ \\
\hline 5 & $5(19.2)$ \\
\hline 6 & $1(3.8)$ \\
\hline 7 & $36.7(3.9-132.5)$ \\
VIT initiation (mo) & \\
\hline
\end{tabular}

VIT, vincristine, irinotecan and temozolomide combination. ${ }^{a}$ High grade sarcoma with mixture of rhabdomyosarcoma and liposarcoma.

ual clinical status.

Toxicity was appraised after each course. All toxic events were graded according to the Common Toxicity Criteria for Adverse Events ver. 4. OS was defined as time interval between the VIT start date and the date of death or last follow-up. Progression-free survival (PFS) was defined as time interval from the VIT start date to the date of disease progression or death. Time to VIT failure (TTF) was determined by time interval between the VIT start date and the date of VIT withdrawal.

\section{Statistical analysis}

Statistical analyses were made using either SPSS ver. 19 (SPSS Inc., Chicago, IL) or Stata/IC ver. 12.0 (StataCorp LP, College Station, TX). Statistical significance was determined at the $\mathrm{p}<0.05$ level. The Kaplan-Meier method was used 
Table 2. VIT response

\begin{tabular}{|c|c|c|c|c|c|}
\hline Disease & $\begin{array}{c}\text { No. of } \\
\text { patients }\end{array}$ & $\begin{array}{l}\text { Median number of } \\
\text { VIT courses (range) }\end{array}$ & $\begin{array}{l}\text { Best response } \\
\qquad\left(\mathbf{N}^{\mathrm{a})}\right)\end{array}$ & $\begin{array}{c}\text { Objective response } \\
\text { rate }^{\text {b) }}(\%)\end{array}$ & $\begin{array}{r}\text { Control } \\
\text { rate }^{c)}(\%)\end{array}$ \\
\hline Rhabdomyosarcoma & 8 & $2(1-6)$ & SD (4), PD (4) & 0 & 50 \\
\hline Osteosarcoma & 7 & $5(1-16)$ & PR (1), SD (2), PD (4) & 14.3 & 42.9 \\
\hline Ewing sarcoma & 3 & $18(1-18)$ & PR (1), PD (1), N/E (1) & 33.3 & 50 \\
\hline Synovial sarcoma & 3 & $7.5(3-12)$ & SD (1) PD (2) & 33.3 & 33.3 \\
\hline Alveolar soft part sarcoma & 2 & $6.5(5-8)$ & $\mathrm{SD}(2)$ & 0 & 100 \\
\hline Hemangiopericytoma & 1 & $5(5)$ & $\mathrm{SD}(1)$ & 0 & 100 \\
\hline Desmoplastic small round cell tumor & 1 & $3(3)$ & $\mathrm{PD}(1)$ & 0 & 0 \\
\hline High grade sarcoma $^{\text {d) }}$ & 1 & $3(3)$ & $\mathrm{SD}(1)$ & 0 & 100 \\
\hline
\end{tabular}

$\mathrm{CR}$, complete remission; $\mathrm{PD}$, progressive disease; $\mathrm{PR}$, partial response; $\mathrm{SD}$, stable disease; VIT, vincristine, irinotecan and temozolomide combination. ${ }^{\mathrm{a})}$ Number of patients, ${ }^{\mathrm{b})}$ Objective response rate; $(\mathrm{CR}+\mathrm{PR}) /$ all $(\%),{ }^{\mathrm{c})}$ Control rate; $(\mathrm{CR}+\mathrm{PR}+\mathrm{SD}) /$ all $(\%)$, ${ }^{\mathrm{d})} \mathrm{High}$ grade sarcoma with mixture of rhabdomyosarcoma and liposarcoma.
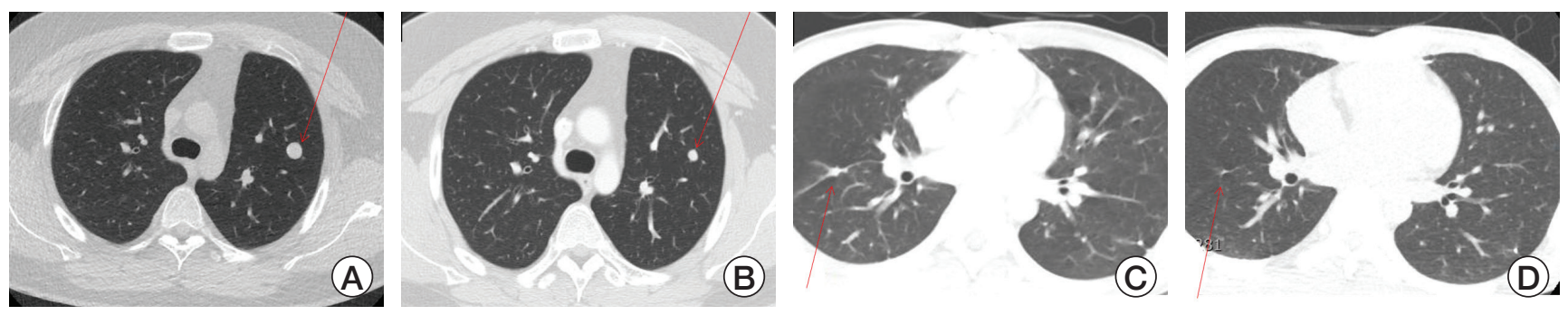

Fig. 1. Pulmonary computed tomography image of two patients who showed partial response. Size change of single metastatic nodule (arrow) before VIT (vincristine, irinotecan and temozolomide combination) treatment (A) and after two courses of VIT treatment (B) in an osteosarcoma patient. Size change of a metastatic nodule (arrow) before VIT treatment (C) and after two courses of VIT treatment (D) in an Ewing sarcoma patient.

to estimate survival rates, and Cox regression analysis was done to evaluate the relation between clinical factors and survival.

\section{Results}

\section{Patients and disease characteristics}

Twenty-six patients (12 males, 14 females) were identified in the study (Table 1). Median age at initial diagnosis was 14.9 years (range, 1.0 to 35.2 years), and median age at starting VIT was 18.5 years (range, 2.0 to 39.9 years). The most common diagnosis was rhabdomyosarcoma (8 patients, $30.8 \%$ ), followed by osteosarcoma (7 patients, 26.9\%). Twenty-five patients had gross tumor when starting VIT. VIT was delivered as 2nd to 7th line of treatment, with 4th most common. Number of VIT courses was 1-18, with two most common (6 of 26, 23.1\%). With respect to local control measure, two patients with PR underwent surgical resection on VIT therapy, while nine patients with SD or progressive disease (PD) after withdrawal. As for radiotherapy, one patient received radiation on VIT therapy, and six after VIT withdrawal.

\section{Treatment response}

Excluding one patient with Ewing sarcoma who underwent surgery for lung metastasis and subsequently achieved a complete remission (CR) before VIT therapy, 25 patients were evaluable for best treatment response (Table 2). Of the 25, two partial response (PR), 11 SD, and 12 PD were observed with an objective response rate $(\mathrm{CR}+\mathrm{PR})$ and a control rate $(\mathrm{CR}+\mathrm{PR}+\mathrm{SD})$ of $8 \%$ and $52 \%$, respectively.

As for two patients with PR, one had osteosarcoma in the lung. He was initially diagnosed with osteosarcoma at age 17 , and underwent methotrexate, doxorubicin, and cisplatin chemotherapy and surgery for the tumor of left proximal tibia. Two years later, he presented with metastatic relapse in the lung. ICE and TC had been sequentially applied, but with two more relapse. Finally, he received VIT as the fourth line. PR was obtained after two VIT courses (Fig. 1A and B), and reduced lung nodule was subsequently excised. He 


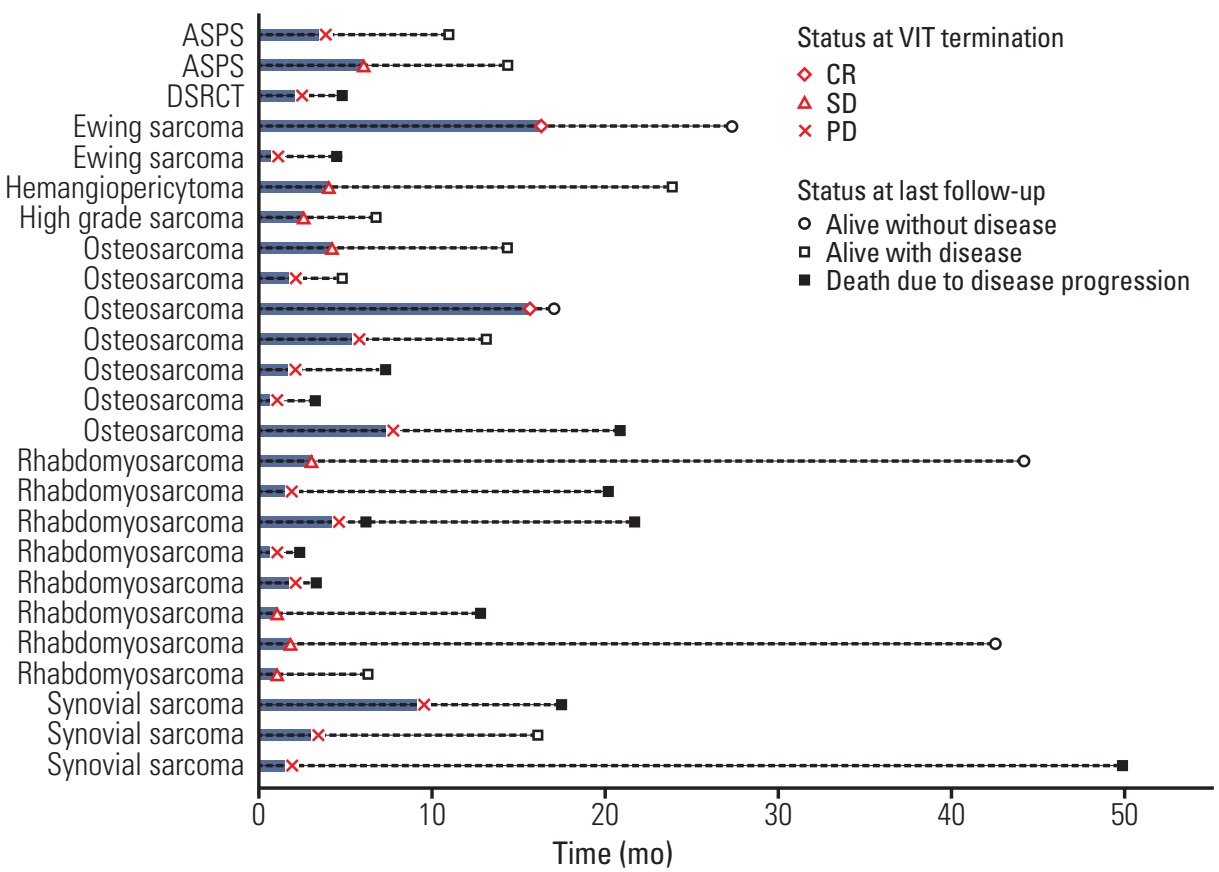

Fig. 2. Time to VIT-failure and subsequent outcome. ASPS, alveolar soft-part sarcoma; CR, complete remission; DSRCT, desmoplastic small round cell tumor; PD, progressive disease; SD, stable disease; VIT, vincristine, irinotecan and temozolomide combination.

received additional 14 VIT courses and remained diseasefree for 3 months off-therapy.

Another 13-year-old boy had pelvic Ewing sarcoma with lung metastasis at initial diagnosis. He was given vincristine, ifosfamide, doxorubicin, and etoposide combination followed by hemipelvectomy and excision of lung nodules. On progression of metastatic lung disease, he had taken vincristine, doxorubicin, and ifosfamide combination, and then $\mathrm{TC}$, showing no response. Ultimately, he adopted VIT as the fourth line. After two courses of VIT, metastatic lung nodules were decreased, fulfilling PR criteria (Fig. 1C and D). Then shrunk nodule was surgically removed. After finishing total of 18 VIT courses, he was disease-free during the follow-up period of 12 months.

Two interesting cases need to be mentioned. A patient with Ewing sarcoma was excluded from response evaluation. As described above, the reason for exclusion was that he had taken lung metastatectomy with the result of no measurable lesion just before starting VIT as fifth line. He had been heavily pretreated with various chemotherapeutic regimens, surgery, and radiotherapy for the tumor in the pelvic retroperitoneum only to recur or progress. He remained in CR for more than 6 years after finishing 18 courses of VIT which was started in the state of no measurable lesion in the lung.

A boy with rhabdomyosarcoma displayed a discrepancy between image and histologic findings. For the gross tumor of the prostate and perirectal area measuring $4.6 \mathrm{~cm} \times 3.5$ $\mathrm{cm} \times 4.4 \mathrm{~cm}$ even after delivery of two chemotherapeutic regimens, he had received two courses of VIT without any change in tumor size (SD). Then, his tumor specimen was obtained with core biopsy. Contrary to our expectation, copious amount of the biopsy specimen did not harbor any viable portion histologically. After completion of third course, however, further VIT was abandoned because he presented with uncontrollable vomiting after taking temozolomide even with intense antiemetic therapy. Thus, his chemotherapeutic regimen was changed to TC. After finishing seven courses of TC, he remains in CR for 3.5 years.

VIT response and ultimate outcome of the 25 patients are depicted in Fig. 2. Although PR rate of $8 \%$ was not satisfactory, disease control was achieved in a significant portion (13 of 25, 52\%) (Table 2). For patients with SD or PD on VIT, other diverse chemotherapeutic regimens were substituted for VIT, but none achieved an objective response thereafter.

We explored clinical parameters related to treatment response. Those like sex, disease type, number of recurrences before VIT therapy, and involved sites at the beginning of VIT were not associated with treatment response. $(p=0.317$, $\mathrm{p}=0.716, \mathrm{p}=0.319$, and $\mathrm{p}=0.973$, respectively).

\section{Survival}

With a median follow-up of 23.8 months (range, 2.3 to 71.4 months), for the 25 evaluable patients, four patients were alive without disease; nine with disease; 12 died of disease 

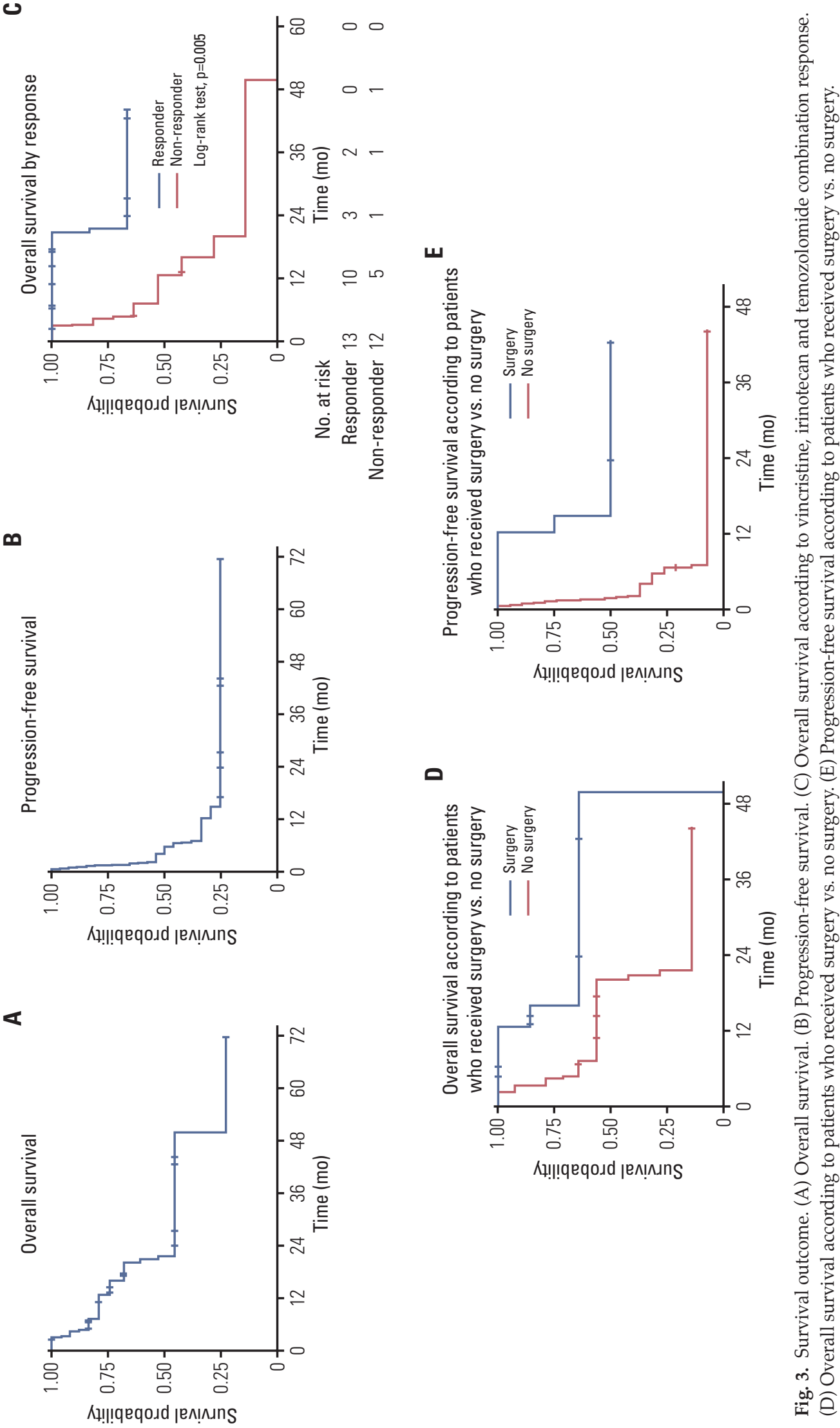
Table 3. Grade II-IV toxicities related to VIT

\begin{tabular}{|c|c|c|c|}
\hline Toxicity $^{a}$ & Grade $2(n=12)$ & Grade $3(n=2)$ & Grade $4(n=1)$ \\
\hline \multicolumn{4}{|c|}{ Non-hematologic toxicity } \\
\hline Diarrhea & 8 & 0 & 0 \\
\hline Nausea/Vomiting & 2 & 1 & 0 \\
\hline Abdominal pain & 1 & 0 & 0 \\
\hline Gastritis & 1 & 0 & 0 \\
\hline \multicolumn{4}{|l|}{ Hematologic toxicity } \\
\hline Thrombocytopenia & 0 & 0 & 1 \\
\hline Neutropenia & 1 & 0 & 0 \\
\hline Neutropenic fever & 0 & 1 & 0 \\
\hline
\end{tabular}

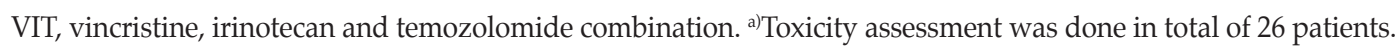

progression. OS rate was 79.3\% (95\% confidence interval [CI], 57.1 to 90.9) at 1 year and $45.5 \%$ (95\% CI, 20.6 to 67.5$)$ at 2 years (Fig. 3A). Clinical variables, such as sex, disease type, number of recurrences or progression before starting VIT, VIT treatment line, and the presence of metastases at the start of VIT were not associated with survival ( $p=0.702$, $p=0.485, p=0.071, p=0.147$, and $p=0.975$, respectively). On the contrary, patients with PR or SD had better survival $(p=0.005)$ (Fig. 3C). Of the 13 patients with $\geq S D$, two died of disease progression and 11 were alive at the last follow-up. In contrast, only two of the 12 patients with PD on VIT were alive at the last follow-up. PFS rate was 33.9\% (95\% CI, 16.7 to 52.0 ) at 1 year and $25.4 \%$ (95\% CI, 10.6 to 43.3 ) at 2 years (Fig. 3B). TTF and outcome at the last follow-up of an individual patient is depicted (Fig. 2). In addition to two patients with PR who were alive without disease at the last follow-up, another two patients with SD remained in CR. One rhabdomyosarcoma patient (rhabdomyosarcoma 15 in Fig. 2) who displayed a discrepancy between image and histologic findings is described above. Another rhabdomyosarcoma patient (rhabdomyosarcoma 21 in Fig. 2) had received surgical resection and chemotherapy for the tumor in the gluteus medius, but subsequently developed metastatic lung relapse. Two courses of VIT were administered with no change in the lung lesion (SD). Thus, metastatectomy was attempted for the first time with complete removal of the lung lesion (CR).

When two patients with PR on VIT were excluded from analysis, patients who underwent tumor resection showed superior PFS $(\mathrm{p}=0.011)$ and a trend toward better OS $(\mathrm{p}=0.057)$ compared with those who did not (Fig. 3D and $\mathrm{E})$. Radiotherapy did not affect survival probability (data not shown).

\section{Toxicity}

The most common toxicity was grade 2 diarrhea which was observed in eight of the 26 patients (30.8\%) (Table 3).
Other non-hematologic toxicities were nausea and / or vomiting in three patients (two grade 2 and one grade 3), grade 2 abdominal pain, grade 2 gastritis, grade 3 colitis, and grade 2 pulmonary hemorrhage in one for each. Pulmonary hemorrhage was thought to be tumor bleeding and was resolved with supportive care alone. Any grade 4 non-hematologic toxicities were not observed. With regards to hematological toxicities, grade 4 thrombocytopenia and grade 3 neutropenic fever was demonstrated in one patient for each. There was no treatment-related death.

\section{Discussion}

Herein, we demonstrate that VIT administered as a salvage regimen was effective in tumor control and tolerable in various types of relapsed or refractory sarcomas. Although the number of subjects in each disease type was quite small, VIT achieved more than $50 \%$ control rate. VIT trials in other studies have been attempted mostly in rhabdomyosarcoma and Ewing sarcoma $[11,12,16]$. Only anecdotal data testing VIT (or IT) in osteosarcoma, synovial sarcoma, alveolar soft part sarcoma, and desmoplastic small round cell tumor are available. Furthermore, we could not find any VIT data on hemangiopericytoma, and mixed rhabdomyosarcoma and liposarcoma. SD was noted in two diseases thus, we could raise the possibility that VIT application might be expanded to more diverse sarcoma types than the ones ever reported. One $(50 \%)$ of the two patients with Ewing sarcoma and one (14.3\%) of the seven patients with osteosarcoma showed PR. Although exempted from response evaluation due to lack of visible tumor (excision of metastatic lung tumor before starting VIT), the Ewing sarcoma patient described above had been heavily pretreated with four regimens and two times of pulmonary metastatectomy before he finally received VIT. Thus, it is possible to speculate that VIT might have contrib- 
uted to his survival without disease over 6 years, considering that two times of previous pulmonary metastatectomy alone had not eradicated the disease. Benefit of VIT in Ewing sarcoma has been demonstrated in other studies as well [11]. Interestingly, the patient with rhabdomyosarcoma who displayed discrepancy between radiologic image and histologic findings described earlier is alive without disease for more than 3.5 years off-therapy. Clinical variables, such as disease type, number of recurrences before VIT treatment, and sites of tumor at the beginning of VIT could not predict VIT response.

OS at 1 year and 2 years were $79.3 \%$ (95\% CI, 57.1 to 90.9) and $45.5 \%$ (95\% CI, 20.6 to 67.5), respectively, and PFS at 1 year and two years were $33.9 \%$ (95\% CI, 16.7 to 52.0 ) and $25.4 \%$ (95\% CI, 10.6 to 43.3 ), respectively (Fig. 3A and B). One-year and two-year OS in Ewing sarcoma patients were $55 \%$ [17] and 25.9\% [10] in other series. PFS varied from $23 \%$ to $49 \%$ depending on the studies $[16,17]$. However, comparison on outcomes between our study and other series is not plausible as patient population was quite different.

Most of the patients in our study were heavily pretreated, and VIT was delivered as a median of 4 th line (range, 2 to 7). Our median value of VIT line was greater than that of any other studies employing irinotecan and temozolomide with or without vincristine in sarcomas $[9,12,16,17]$. In fact, VIT was adopted as 3rd and 4th line in our patients with PR. This suggests that certain proportion of sarcoma patients with multiple relapses or primary refractoriness still have a chance for prolonged survival or even cure, even though VIT is administered after several times of treatment failure.

Clinical variables, such as sex, disease type, number of relapses or progression before starting VIT, number of previous regimens, and the presence of metastases at the start of VIT did not predict outcome. On the contrary, VIT response was associated with outcome, which is consistent with the report on Ewing sarcoma [17]. Besides VIT response, beneficial effect of surgery on survival was examined in our subjects, excluding two patients with PR on VIT from analysis. Patients who had surgical resection fare better in terms of survival. (Fig. 3D and E) This suggests that surgery needs to be considered whenever possible even in patients who do not respond to VIT.

In previous studies, irinotecan and temozolomide with or without vincristine have been shown to be effective most commonly in Ewing sarcoma [8-10,12,17,18]. In agreement with this, PR was observed in one of the two evaluable patients with Ewing sarcoma in our series. In addition, we raise the possibility that VIT was effective in the non-evaluable patient with Ewing sarcoma who underwent VIT after metastectomy and maintained long-term CR afterwards. Another PR was seen in one of the seven patients with osteo- sarcoma. Studies on VIT activity in osteosarcoma are quite limited, and objective response was reported in the range between $25 \%$ (one of the four patients) and $50 \%$ (one of the two patients) $[8,19,20]$. Although objective response rate in our osteosarcoma patients was $14.2 \%$, it is hard to make any comparison since number of subjects in the literature is too small.

In the current study, we included patients with synovial sarcoma, alveolar soft part sarcoma, hemangiopericytoma, desmoplastic small round cell tumor, and mixed rhabdomyosarcoma and liposarcoma. Interestingly, SD was observed in all of those with exception of desmoplastic small round cell tumor. This suggests that VIT might be useful across various sarcoma histology. VIT trial for synovial sarcoma [20] and desmoplastic small round cell tumor [19] is available in only a couple of studies, and that for hemangiopericytoma and mixed rhabdomyosarcoma and liposarcoma has not been reported yet.

As was predicted, gastrointestinal toxicities were most common. Grade 2 diarrhea occurred in $30.8 \%$ of the patients, although cefixime was prescribed prophylactically. However, it was manageable with loperamide with or without atropine prescription. With regards to grade 3 or higher toxicity, grade 3 nausea and / or vomiting, grade 3 colitis, grade 3 neutropenic fever, and grade 4 thrombocytopenia was demonstrated in one patient $(3.8 \%)$ for each. There was no life-threatening morbidity or treatment-related mortality. In a study on Ewing sarcoma [10], grade 3 or higher diarrhea occurred in $13.6 \%$, and grade 3 or higher neutropenia and thrombocytopenia occurred in $4.5 \%$ for each. In another study on Ewing sarcoma [17], grade 3 or higher diarrhea, neutropenia, thrombocytopenia, and nausea and/or vomiting developed in $4 \%, 12 \%, 4 \%$, and $2 \%$, respectively. Compared with these studies, incidence rate of grade 3 or higher toxicities was comparable; however, $\geq$ grade 3 diarrhea was not reported in our series. Vigorous prescription of antidiarrheals with prophylactic cefixime might have played a role.

Our results have some limitations. First, it is a retrospective analysis based on small sample size. Second, incidence of actual toxicities could have been reported less as data were collected retrospectively. However, we believe that at least grade 3 or higher toxicities were not likely to be missed.

The current study has several implications. First, various sarcoma histology, such as rhabdomyosarcoma, osteosarcoma, Ewing sarcoma, synovial sarcoma, alveolar soft part sarcoma, hemangiopericytoma, desmoplastic small round cell tumor, and mixed rhabdomyosarcoma and liposarcoma were included in the study. To our knowledge, there has been no report on VIT trial for hemangiopericytoma and mixed rhabdomyosarcoma and liposarcoma, and only a couple of reports for synovial sarcoma and alveolar soft part sarcoma. 
In addition, SD was observed in these four sarcoma types, suggesting that VIT as a palliative regimen might be utilized broadly across various sarcoma histology. Second, VIT was administered in heavily pretreated patients considering that our median VIT line was fourth, the highest value ever reported. Nonetheless, we could obtain 52\% control rate. Moreover, the regimen was tolerable enough that only one of the 26 patients refused to take the regimen in the middle due to grade 3 nausea and vomiting. One plausible reason for low toxicity is low hematologic toxicity of VIT regimen. Taken these together, we argue that VIT may remain an option even when there is little hope for further treatment in a far advanced sarcoma.

Conclusively, we demonstrate that the VIT regimen was active and tolerable in our cohort with a variety of relapsed and/or refractory sarcoma. However, even with our meaningful VIT response, long-term survival is still not satisfactory. Therefore, further efforts need to be pursed such as, VIT combined with targeted or immune-oncologic agents at least in certain histologic types of sarcoma.

In conclusion, with the VIT regimen delivered in 26 recurrent and / or refractory patients with various sarcoma histology, we could achieve $8 \%$ PR and 52\% control rate. OS and PFS rates were $79.3 \%$ and $33.9 \%$ at 1 year, and $45.5 \%$ and $25.4 \%$ at 2 years, respectively. Even though the regimen was given at a late stage of treatment with a median line of 4 th, it was quite tolerable. Grade 3 and grade 4 toxicity was observed in $11.5 \%$ and $3.8 \%$ of our cohort, respectively, and serious treatmentrelated morbidity or mortality did not occur. Thus, the VIT regimen was active and tolerable in our recurrent and/or refractory sarcoma patients with various histology.

\section{Ethical Statement}

The study was approved by the Institutional Review Board (IRB) of the NCC (IRB No. NCC2019-0141), and the requirement for informed consent was waived because of the retrospective nature of the study.

\section{Author Contributions}

Conceived and designed the analysis: Park BK.

Collected the data: Ju HY.

Contributed data or analysis tools: Park M, Lee JA, Park HJ, Park SY, Kim JH, Kang HG, Yang HC, Park BK.

Performed the analysis: Ju HY.

Wrote the paper: Ju HY.

\section{Conflicts of Interest}

Conflict of interest relevant to this article was not reported.

\section{Acknowledgments}

This work was supported by the Korean Association for Children with Leukemia and Cancer (KACLC; 1841930-1).

\section{References}

1. Linabery AM, Ross JA. Trends in childhood cancer incidence in the U.S. (1992-2004). Cancer. 2008;112:416-32.

2. Spunt SL, Pappo AS. Childhood nonrhabdomyosarcoma soft tissue sarcomas are not adult-type tumors. J Clin Oncol. 2006;24:1958-9.

3. Ou JY, Spraker-Perlman H, Dietz AC, Smits-Seemann RR, Kaul S, Kirchhoff AC. Conditional survival of pediatric, adolescent, and young adult soft tissue sarcoma and bone tumor patients. Cancer Epidemiol. 2017;50:150-7.

4. Park HJ, Moon EK, Yoon JY, Oh CM, Jung KW, Park BK, et al. Incidence and survival of childhood cancer in Korea. Cancer Res Treat. 2016;48:869-82.

5. Van Winkle P, Angiolillo A, Krailo M, Cheung YK, Anderson $\mathrm{B}$, Davenport $\mathrm{V}$, et al. Ifosfamide, carboplatin, and etoposide (ICE) reinduction chemotherapy in a large cohort of children and adolescents with recurrent/refractory sarcoma: the Children's Cancer Group (CCG) experience. Pediatr Blood Cancer. 2005;44:338-47.

6. Saylors RL 3rd, Stine KC, Sullivan J, Kepner JL, Wall DA, Bernstein ML, et al. Cyclophosphamide plus topotecan in children with recurrent or refractory solid tumors: a Pediatric Oncology Group phase II study. J Clin Oncol. 2001;19:3463-9.
7. Rapkin L, Qayed M, Brill P, Martin M, Clark D, George BA, et al. Gemcitabine and docetaxel (GEMDOX) for the treatment of relapsed and refractory pediatric sarcomas. Pediatr Blood Cancer. 2012;59:854-8.

8. Buyukkapu Bay S, Kebudi R, Gorgun O, Zulfikar B, Darendeliler E, Cakir FB. Vincristine, irinotecan, and temozolomide treatment for refractory/relapsed pediatric solid tumors: a single center experience. J Oncol Pharm Pract. 2019;25:1343-8.

9. Kurucu N, Sari N, Ilhan IE. Irinotecan and temozolamide treatment for relapsed Ewing sarcoma: a single-center experience and review of the literature. Pediatr Hematol Oncol. 2015;32:50-9.

10. Raciborska A, Bilska K, Drabko K, Chaber R, Pogorzala M, Wyrobek E, et al. Vincristine, irinotecan, and temozolomide in patients with relapsed and refractory Ewing sarcoma. Pediatr Blood Cancer. 2013;60:1621-5.

11. Wagner L. Camptothecin-based regimens for treatment of ewing sarcoma: past studies and future directions. Sarcoma. 2011;2011:957957.

12. Wagner LM, McAllister N, Goldsby RE, Rausen AR, McNallKnapp RY, McCarville MB, et al. Temozolomide and intravenous irinotecan for treatment of advanced Ewing sarcoma. 
Pediatr Blood Cancer. 2007;48:132-9.

13. Pappo AS, Lyden E, Breitfeld P, Donaldson SS, Wiener E, Parham D, et al. Two consecutive phase II window trials of irinotecan alone or in combination with vincristine for the treatment of metastatic rhabdomyosarcoma: the Children's Oncology Group. J Clin Oncol. 2007;25:362-9.

14. Furman WL, Crews KR, Billups C, Wu J, Gajjar AJ, Daw NC, et al. Cefixime allows greater dose escalation of oral irinotecan: a phase I study in pediatric patients with refractory solid tumors. J Clin Oncol. 2006;24:563-70.

15. Nishino M, Jagannathan JP, Ramaiya NH, Van den Abbeele AD. Revised RECIST guideline version 1.1: What oncologists want to know and what radiologists need to know. AJR Am J Roentgenol. 2010;195:281-9.

16. Setty BA, Stanek JR, Mascarenhas L, Miller A, Bagatell R, Okcu F, et al. VIncristine, irinotecan, and temozolomide in children and adolescents with relapsed rhabdomyosarcoma. Pediatr Blood Cancer. 2018;65:e26728.
17. Palmerini E, Jones RL, Setola E, Picci P, Marchesi E, Luksch R, et al. Irinotecan and temozolomide in recurrent Ewing sarcoma: an analysis in 51 adult and pediatric patients. Acta Oncol. 2018;57:958-64.

18. Casey DA, Wexler LH, Merchant MS, Chou AJ, Merola PR, Price AP, et al. Irinotecan and temozolomide for Ewing sarcoma: the Memorial Sloan-Kettering experience. Pediatr Blood Cancer. 2009;53:1029-34.

19. McNall-Knapp RY, Williams CN, Reeves EN, Heideman RL, Meyer WH. Extended phase I evaluation of vincristine, irinotecan, temozolomide, and antibiotic in children with refractory solid tumors. Pediatr Blood Cancer. 2010;54:909-15.

20. Wagner LM, Perentesis JP, Reid JM, Ames MM, Safgren SL, Nelson MD Jr, et al. Phase I trial of two schedules of vincristine, oral irinotecan, and temozolomide (VOIT) for children with relapsed or refractory solid tumors: a Children's Oncology Group phase I consortium study. Pediatr Blood Cancer. 2010;54:538-45. 